\title{
Genetic variance and plasma concentration of CD93 is associated with cardiovascular mortality: Results from a 6.7-year follow-up of a healthy community-living elderly population
}

\author{
URBAN ALEHAGEN $^{1}$, LEVAR SHAMOUN ${ }^{2,3}$ and DICK WÅGSÄTER ${ }^{3}$ \\ ${ }^{1}$ Division of Cardiovascular Medicine, Department of Health, Medicine and Caring Sciences, \\ Faculty of Medicine, Linköping University, SE-581 85 Linköping; ${ }^{2}$ Division of Medical Diagnostics, \\ Department of Laboratory Medicine, Jönköping County, SE-553 05 Jönköping; \\ ${ }^{3}$ Department of Medical Cell Biology, Uppsala University, SE-752 36 Uppsala, Sweden
}

Received June 25, 2020; Accepted September 3, 2020

DOI: $10.3892 / \mathrm{mmr} .2020 .11555$

\begin{abstract}
Inflammation is one of the fundamental processes in numerous diseases. Cluster of differentiation (CD) 93, a glycoprotein, has been reported to be associated with a number of these diseases. There are reports indicating that a high plasma level of CD93 is associated with adverse events in ischaemic heart disease. Additionally, there are reports indicating different cardiovascular risks between different single nucleotide polymorphisms (SNPs) of CD93. Therefore, the present study aimed to determine whether the plasma concentration of CD93 and polymorphism of rs2749812 in CD93 were associated with clinical conditions and mortality in an elderly population. In 470 healthy elderly community-living individuals a novel clinical examination involving echocardiography and blood sampling was performed. The population was followed for 6.7 years. Plasma levels of CD93 and SNP analyses of rs2749812 of CD93 using PCR methodology were used. During the follow-up period, $106(22.6 \%)$ all-cause and $61(13.0 \%)$ cardiovascular deaths were registered. Those with the highest plasma concentration had markedly higher all-cause mortality. Evaluating the A/A, A/G and G/G genotypes, the G/G group exhibited significantly higher cardiovascular mortality $(\mathrm{P}=0.026)$, and an almost two-fold increased risk in a multivariate Cox regression model compared with the $\mathrm{A} / \mathrm{G}$ genotype. Evaluation of subgroups with respect to sex, diabetes
\end{abstract}

Correspondence to: Professor Urban Alehagen, Division of Cardiovascular Medicine, Department of Health, Medicine and Caring Sciences, Faculty of Medicine, Linköping University, SE-581 85 Linköping, Sweden

E-mail: urban.alehagen@liu.se

Abbreviations: CI, confidence interval; CV, cardiovascular; ECG, electrocardiogram; EF, ejection fraction; HR, hazard ratio; SD, standard deviation

Key words: CD93, genotypes, elderly, mortality and hypertension revealed markedly increased cardiovascular risk in the $\mathrm{G} / \mathrm{G}$ genotype in all subgroups. All results persisted in the multiple models used. In the present study, the glycoprotein CD93 was demonstrated to have prognostic cardiovascular information, with increased risk for those with a high plasma concentration. Furthermore, the G/G genotype of rs2749812 of CD93 has a significantly higher cardiovascular risk, as demonstrated here, and could therefore be regarded as a possible cardiovascular risk biomarker that might in the future be used to offer optimised cardiovascular patient handling. However, this was a small study, and more research is required.

\section{Introduction}

Cluster of differentiation (CD) 93 is a transmembrane glycoprotein that is expressed in monocytes, leukocytes and endothelial cells (1-3). There are reports indicating an association between CD93 and diseases of which inflammation is a fundamental characteristic, such as asthma bronchitis $(4,5)$, and arthritis (3). As psoriasis is characterised by chronic inflammation, the level of CD93 has been shown to increase in psoriatic skin lesions (6). It has also been reported that in patients with colorectal cancer, the level of CD93 is higher compared to a normal reference population (7). An important association between CD93 and angiogenesis that might influence tumour growth has been discussed in the literature (8). In an interesting review by Khan et al (9), the authors also discuss the relationship between CD93 and thrombomodulin, which has a central position in the coagulation cascade. As CD93 and thrombomodulin belong to the same transmembrane glycoprotein family, it is not surprising to note the effects of thrombomodulin on inflammation, which are similar to the effects influenced by CD93.

Cardiovascular diseases are among the most important disease groups that occur in the Western population (10), with a high mortality. Inflammation is one of the cornerstones in the development of ischaemic heart disease (11-14). There has been information indicating that single nucleotide polymorphisms (SNPs) from CD93 have an association with ischaemic heart disease; van der Net et al (15) showed a $26 \%$ increased 
risk for ischaemic heart disease with one specific polymorphism of rs3746731 of CD93.

A soluble form of CD93 has been demonstrated (1), and Youn et al (16) reported increased levels of this soluble form of CD93 in plasma from patients with myocardial infarction, but also an association with adverse events and mortality in those patients. This relation is important in the risk evaluation of patients with ischaemic heart disease. However, in the report from Youn et al (16), the sample sizes of the group suffering from mortality were extremely small (all-cause mortality: $\mathrm{n}=18$; cardiovascular mortality; $\mathrm{n}=9)$; thus, the clinical information is uncertain. Mälarstig et al (17) showed that the polymorphisms of $r s 2749812$ are associated with CD93 gene expression level in human peripheral blood mononuclear cells (PBMCs).

Therefore, the aim of this study was to determine whether the plasma concentration of CD93 and the polymorphism of rs2749812 in CD93 were associated with well-known clinical conditions, and mortality.

\section{Materials and methods}

Patient population. From a rural municipality in the south-east of Sweden, a population of 470 individuals (men: 239, 50.9\%; females: 230, 48.9\%) with a median age of 76 years (interquartile range: 18 years) were included in the study. All were part of a longitudinal epidemiological project focusing on cardiovascular risk factors among the elderly (18). The participants in that project were invited to participate in the present sub-study, with the first patient included on the 13th of January 2003, and the last on the 28 th of June 2005. The inclusion criteria included all those living in a rural municipality aged between 70 and 85 years. This was in order to minimise bias in the selection process. The population that agreed to participate delivered blood samples, and were given echocardiographic examinations and an electrocardiogram (ECG). The New York Heart Association functional class was evaluated by the including physician based on the patient information.

All participants gave their written informed consent, and the study was conducted in accordance with the Declaration of Helsinki principles. The study protocol was approved by the Regional Ethical Review Board of Linköping, Sweden (Dnr 95044). The mortality information was obtained from autopsy reports or from the National Board of Health and Welfare in Sweden, which registers all deaths.

Comorbidity. The following definitions have been used in this study; hypertension was defined as a blood pressure of more than 140/90 $\mathrm{mm} \mathrm{Hg}$ measured in the right arm with the patient in the supine position after at least 30 min rest. Hypertension was also assumed if the participant had previously been diagnosed with hypertension and was receiving antihypertensive medication. Diabetes mellitus was defined as a previous diagnosis with on-going treatment, or a fasting blood glucose $\geq 7 \mathrm{mmol} / \mathrm{l}$ measured on one single occasion. Ischaemic heart disease (IHD) was defined as a history of angina pectoris/myocardial infarction or ECG-verified myocardial infarction. Heart failure was defined as a previous diagnosis with on-going treatment, or symptoms/signs of heart failure and objective demonstration of reduced cardiac function in terms of impaired cardiac function on echocardiography. Cardiovascular death was defined as death caused by fatal arrhythmias, myocardial infarction, heart failure, or cerebrovascular insult.

For the methods in general we followed the methods used in our previous publication (19).

Echocardiographic examinations. All echocardiography examinations were performed using an Accuson XP-128c with the patient in a supine, left position. Values for systolic function expressed as left ventricular ejection fraction (EF), were categorised into four classes with interclass limits of 30, 40 and $50 \%$. Normal systolic function was defined as $\mathrm{EF} \geq 50 \%$. Thus, only the systolic function was evaluated.

Determination of CD93 levels in plasma. The blood samples were obtained while the patients were at rest in a supine position, and all samples were collected in pre-chilled plastic Vacutainer tubes (Terumo EDTA K-3). Plasma was prepared by centrifugation at $3,000 \mathrm{x}$ g for $10 \mathrm{~min}$ at $4^{\circ} \mathrm{C}$. All samples were stored at $-70^{\circ} \mathrm{C}$ until analysis. None of the samples was thawed more than twice. Determination of CD93 levels in plasma was performed according to the recommendations (\# DCD930, R\&D Systems Europe, Ltd.) and as previously described (7).

Genotype determination. DNA was isolated from peripheral blood using the QIAmp DNA mini kit (Qiagen $\mathrm{GmbH}$ ), following the manufacturer's instructions. DNA (10 $\mathrm{ng}$ ) was mixed with TaqMan Universal PCR Master mix II and TaqMan SNP probes (Applied Biosystems; Thermo Fisher Scientific, Inc.) and genotypes of SNPs rs2749812 were analysed with the 7500 Fast Real-Time Polymerase Chain Reaction (PCR) system with allelic discrimination according to the protocol used in a previous publication (7).

Statistical analysis. Descriptive data are presented as percentages or mean and standard deviation (SD). Comparative analyses were performed using the Student's unpaired two-sided t-test, whereas the Chi-square test was used for discrete variables, and ANOVA analyses with Bonferroni post hoc tests were performed when evaluating three or more groups with data on a nominal scale-level. Both univariate and multivariate Cox proportional hazard regression analyses were used to analyse the risk of mortality during the follow-up period, where both all-cause mortality and cardiovascular mortality were analysed. Kaplan-Meier evaluation which by definition includes a log-rank test was used to illustrate cardiovascular mortality as a function of follow-up time. Censored patients were those who were still alive at the end of the study period or who had died. Completed patients comprised those who had died. In the multivariate regression model, adjustments were made for the following co-variates: Age, IHD, hypertension, diabetes, New York Heart Association functional class III, angiotensin converting enzyme (ACE) inhibitors/Angiotensin receptor blockers, beta-blockers, diuretics, and $\mathrm{Hb}<120 \mathrm{~g} / 1$.

A P-value $<0.05$ was considered statistically significant. All data were analysed using standard software packages (Statistica v. 13.2, Statsoft Inc, Tulsa, OK, USA). 
Table I. Basal characteristics of the study population divided by genotypes.

\begin{tabular}{|c|c|c|c|c|}
\hline \multirow[b]{2}{*}{ Variable } & \multicolumn{3}{|c|}{ Total population } & \multirow[b]{2}{*}{$\mathrm{P}$-value } \\
\hline & $\mathrm{A} / \mathrm{A}$ & $\mathrm{A} / \mathrm{G}$ & $\mathrm{G} / \mathrm{G}$ & \\
\hline Number & 17 & 152 & 301 & \\
\hline Age (years), mean (SD) & $77.5(3.7)$ & $77.0(3.3)$ & $77.0(3.5)$ & \\
\hline $\mathrm{HT}, \mathrm{n}(\%)$ & $12(70.6)$ & $123(80.9)$ & $219(72.8)$ & 0.15 \\
\hline IHD, n (\%) & $2(11.8)$ & $32(21.1)$ & $68(22.6)$ & 0.56 \\
\hline Diabetes, n (\%) & $4(23.5)$ & $47(30.9)$ & $102(33.9)$ & 0.59 \\
\hline NYHA III, n (\%) & $5(29.4)$ & $31(20.4)$ & 59 (19.6) & 0.62 \\
\hline $\mathrm{AF}, \mathrm{n}(\%)$ & $2(11.8)$ & $15(9.9)$ & $31(10.3)$ & 0.97 \\
\hline $\mathrm{EF}<40 \%, \mathrm{n}(\%)$ & $1(5.9)$ & $7(4.6)$ & $28(9.3)$ & 0.20 \\
\hline ACEI/ARB, n (\%) & $5(39.4)$ & $47(30.9)$ & $69(22.9)$ & 0.17 \\
\hline $\mathrm{BB}, \mathrm{n}(\%)$ & $2(11.8)$ & $63(41.4)$ & $101(33.6)$ & 0.03 \\
\hline Diuretics, n (\%) & $7(41.2)$ & $59(38.8)$ & $106(35.2)$ & 0.70 \\
\hline $\mathrm{Hb}<120 \mathrm{~g} / 1, \mathrm{n}(\%)$ & $0(0.0)$ & $20(13.2)$ & $31(10.3)$ & 0.36 \\
\hline NT-proBNP $(\mu \mathrm{g} / \mathrm{l})$, mean (SD) & $144.9(157.0)$ & $167.6(178.3)$ & $199.2(380.0)$ & 0.54 \\
\hline eGFR $<60 \mathrm{ml} / \mathrm{min}, \mathrm{n}(\%)$ & $7(43.8)$ & $79(52.0)$ & $142(47.2)$ & 0.58 \\
\hline BMI, mean (SD) & $27.6(3.00)$ & $27.2(3.9)$ & $27.2(4.51)$ & 0.91 \\
\hline
\end{tabular}

ACEI, ACE-inhibitors; AF, atrial fibrillation; ARB, angiotensin receptor blockers; BB, beta blockers; BMI, body mass index; EF, ejection fraction according to echocardiography; eGFR, estimated glomerular filtration rate; HT, hypertension; IHD, ischemic heart disease; NT-proBNP, $\mathrm{N}$-terminal fragment of B-type natriuretic peptide.

\section{Results}

Study population characteristics. The basal characteristics of the three genotypes in the total study population, divided into the two sexes, are presented in Table I. From the table, it can be noted that the population was a representative elderly Western population, and it can be seen that in the total study population only a small proportion, $3.6 \%$, had the A/A genotype. As regards the other two genotypes, $32.3 \%$ had the $\mathrm{A} / \mathrm{G}$ genotype, and $64 \%$ had the G/G genotype. Due to the small size of the A/A genotype, considerable uncertainty would appear in statistical evaluations of this genotype in comparison with the other two. We therefore decided not to perform any risk evaluations on those with the A/A genotype, but only on those with the $A / G$ and the $G / G$ genotypes.

From Table I, it can be seen that the population represents an elderly population as generally found in the Western hemisphere, with a majority having hypertension, and thus being treated with ACE inhibitors/angiotensin receptor blockers or beta-blockers, while around $20 \%$ suffered from IHD, and around $30 \%$ suffered from diabetes. No significant differences regarding the basal characteristics could be seen comparing the $\mathrm{A} / \mathrm{G}$ and the $\mathrm{G} / \mathrm{G}$ genotypes.

During the follow-up period of 6.7 years, 106 cases of all-cause mortality (22.6\%), and 61 cases of cardiovascular mortality (13\%) were registered.

Plasma concentration and comorbidity or mortality. In the study population 457 individuals were evaluated regarding the plasma concentration of CD93 rs2749812. The results of the plasma concentration were distributed into quartiles, and clinical variables well-known to influence cardiovascular (CV) prognosis as well as mortality were evaluated in relation to the plasma concentration of CD93 (Table II). From this analysis it could be seen that there was a higher plasma concentration of the cardiac natriuretic peptide NT-proBNP in the fourth quartile $(\mathrm{P}=0.03)$. However, as the number of groups evaluated was high, there was a risk of missing significant differences between two groups when performing significant tests of all groups. We therefore also evaluated the quartiles with the lowest versus the highest plasma concentration of CD93.

From this evaluation, it was demonstrated that in the fourth quartile, significantly more patients with hypertension could be found than in the first quartile $\left(94 / 114 \mathrm{vs} .78 / 115 ; \chi^{2}: 6.55 ; \mathrm{P}=0.011\right)$. Also, there was a borderline difference in the number of those on treatment with ACE inhibitors/Angiotensin II receptor blockers in the fourth quartile, compared to those in the first quartile $(38 / 114$ vs. $\left.25 / 115 ; \chi^{2}: 3.86 ; \mathrm{P}=0.05\right)$. No difference in treatment with beta-blockers could be seen. However, significantly more patients in the fourth quartile were on treatment with diuretics, compared to the first quartile (54/114 vs. $\left.37 / 115 ; \chi^{2}: 5.52 ; \mathrm{P}=0.019\right)$.

Also, evaluating data on renal function revealed a significantly higher s-creatinine level in the fourth quartile of CD93 rs2749812, as compared to those in the first quartile (mean Q1: $87 \mu \mathrm{mol} / 1$ vs. Q4:94 $\mu \mathrm{mol} / \mathrm{l} ; \mathrm{t}=3.70 ; \mathrm{P}=0.0003)$. Use of the Modification of Diet in Renal Disease (MDRD) Study equation for renal function, an estimated glomerular function model, showed a higher estimated glomerular function for those in the first quartile, as compared to those in the fourth quartile $(71.8 \mathrm{ml} / \mathrm{min}$ vs. $66.0 \mathrm{ml} / \mathrm{min}$; $\mathrm{t}=3.46 ; \mathrm{P}=0.0006)$.

The total mortality over 6.7 years distributed into several groups is presented in Table III. From that, it can be seen that even if a numerical difference in CV mortality between those in the first and fourth quartiles is apparent; no significant 
Table II. Basal characteristics of the study population divided into quartiles according to plasma concentration of IL-32.

\begin{tabular}{|c|c|c|c|c|c|}
\hline \multirow[b]{2}{*}{ Variable } & \multicolumn{4}{|c|}{ Total population } & \multirow[b]{2}{*}{ P-value } \\
\hline & Q1 & Q2 & Q3 & Q4 & \\
\hline Number & 115 & 114 & 114 & 114 & \\
\hline Age (years), mean (SD) & $77.5(3.7)$ & $77.0(3.3)$ & $77.0(3.5)$ & $76.8(3.4)$ & \\
\hline HT, n (\%) & $12(70.6)$ & $123(80.9)$ & $219(72.8)$ & $94(82.5)$ & 0.15 \\
\hline IHD, n (\%) & $2(11.8)$ & $32(21.1)$ & $68(22.6)$ & $26(22.8)$ & 0.56 \\
\hline Diabetes, n (\%) & $4(23.5)$ & $47(30.9)$ & $102(33.9)$ & $36(31.6)$ & 0.59 \\
\hline NYHA III, n (\%) & $5(29.4)$ & $31(20.4)$ & $59(19.6)$ & $22(19.3)$ & 0.62 \\
\hline $\mathrm{AF}, \mathrm{n}(\%)$ & $2(11.8)$ & $15(9.9)$ & $31(10.3)$ & $9(7.9)$ & 0.97 \\
\hline $\mathrm{EF}<40 \%, \mathrm{n}(\%)$ & $1(5.9)$ & $7(4.6)$ & $28(9.3)$ & $9(7.9)$ & 0.20 \\
\hline ACEI/ARB, n (\%) & $5(39.4)$ & $47(30.9)$ & $69(22.9)$ & $38(33.3)$ & 0.17 \\
\hline $\mathrm{BB}, \mathrm{n}(\%)$ & $2(11.8)$ & $63(41.4)$ & $101(33.6)$ & $47(41.2)$ & 0.03 \\
\hline Diuretics, n (\%) & $7(41.2)$ & $59(38.8)$ & $106(35.2)$ & $54(47.4)$ & 0.70 \\
\hline $\mathrm{Hb}<120$ g/l, n (\%) & $0(0.0)$ & $20(13.2)$ & $31(10.3)$ & $16(14.0)$ & 0.36 \\
\hline NT-proBNP $(\mu \mathrm{g} / \mathrm{l})$, mean $(\mathrm{SD})$ & $144.9(157.0)$ & $167.6(178.3)$ & $199.2(380.0)$ & $261.3(513.2)$ & 0.03 \\
\hline eGFR $<60 \mathrm{ml} / \mathrm{min}, \mathrm{n}(\%)$ & $7(43.8)$ & $79(52.0)$ & $142(47.2)$ & $60(52.6)$ & 0.58 \\
\hline BMI, mean (SD) & $27.6(3.0)$ & $27.2(3.9)$ & $27.2(4.5)$ & $27.0(4.3)$ & 0.91 \\
\hline
\end{tabular}

ACEI, ACE-inhibitors; AF, atrial fibrillation; ARB, angiotensin receptor blockers; BB, beta blockers; BMI, body mass index; EF, ejection fraction according to echocardiography; eGFR, estimated glomerular filtration rate; HT, hypertension; IHD, ischemic heart disease; NT-proBNP, $\mathrm{N}$-terminal fragment of B-type natriuretic peptide.

Table III. Distribution of mortality in the highest vs. the lowest quartile groups of plasma concentration of CD93 in the study population during 6.7 years of follow-up.

\begin{tabular}{lccc}
\hline Variable & Quartile 1 & Quartile 4 & P-value \\
\hline CV-mortality, n & $13 / 114$ & $17 / 114$ & 0.43 \\
Tumors, n & $9 / 114$ & $15 / 114$ & 0.20 \\
Infections, n & 0 & $3 / 114$ & \\
All-cause mortality, n & $24 / 114$ & $38 / 114$ & $\chi^{2}: 4.34 ;$ \\
& & & $P=0.037$
\end{tabular}

$\mathrm{CV}$, cardiovascular.

difference is demonstrated. However, when evaluating the all-cause mortality, a significantly higher mortality can be found in the fourth quartile group, compared with the first quartile group $\left(\chi^{2}: 4.34 ; \mathrm{p}=0.037\right)$.

When applied in a multivariate model, the high plasma concentration of CD93 exhibited independent prognostic information regarding all-cause mortality (HR: 1.91; $\mathrm{P}=0.002$; 95\% CI: 1.28-2.86) Table IV.

The plasma concentration was also evaluated in the three genotypes A/A, A/G and G/G. From this, a significantly higher plasma concentration of CD93 could be found in the $A / G$, compared with the $\mathrm{G} / \mathrm{G}$ genotype $(314 \mathrm{ng} / \mathrm{ml} \mathrm{vs} .285 \mathrm{ng} / \mathrm{ml}$; $\mathrm{T}$ : 2.59; $\mathrm{P}=0.01)$. No significant differences could be seen in the other genotype comparisons. However, by analysing the participants who had a plasma concentration within the fourth quartile, and who had also suffered a CV death $(n=17)$,
Table IV. Cox proportional hazard regression analysis evaluating risk of all-cause mortality in the study population regarding CD93.

\begin{tabular}{lccc}
\hline Variable & $\begin{array}{c}\text { Hazard } \\
\text { ratio }\end{array}$ & $\begin{array}{c}\text { 95\% confidence } \\
\text { interval }\end{array}$ & P-value \\
\hline Diabetes & 1.46 & $0.99-2.17$ & 0.06 \\
Hypertension & 1.21 & $0.76-1.95$ & 0.42 \\
Ischemic heart disease & 1.82 & $1.18-2.79$ & 0.006 \\
eGFR <6 ml/min/1.73 & 1.58 & $1.05-2.36$ & 0.03 \\
ACEI/ARB & 1.00 & $0.66-1.54$ & 0.98 \\
Beta blockers & 0.92 & $0.61-1.39$ & 0.70 \\
CD93 Q4 & 1.91 & $1.28-2.86$ & 0.002 \\
\hline
\end{tabular}

ACEI, ACE-inhibitor; ARB, angiotensin receptor blocker; eGFR, estimated glomerular fraction (by use of the MDRD formula); Q4, 4th quartile.

three out of 17 belonged to the $\mathrm{A} / \mathrm{G}$ genotype, and 13 out of 17 belonged to the G/G genotype. Thus, there was a significantly higher $\mathrm{CV}$ risk for the $\mathrm{G} / \mathrm{G}$ genotype also in the highest quartile of plasma concentration of CD93.

Genotypes and mortality. Mortality was evaluated during the same time span (6.7 years), where both all-cause, and CV mortality were registered. From this evaluation, significant differences in $\mathrm{CV}$ mortality could be demonstrated, but not in all-cause mortality when comparing the two genotypes $\mathrm{A} / \mathrm{G}$ and G/G (CV mortality: A/G: 11/152=7.2\%; G/G: 47/301=15.6\%; 

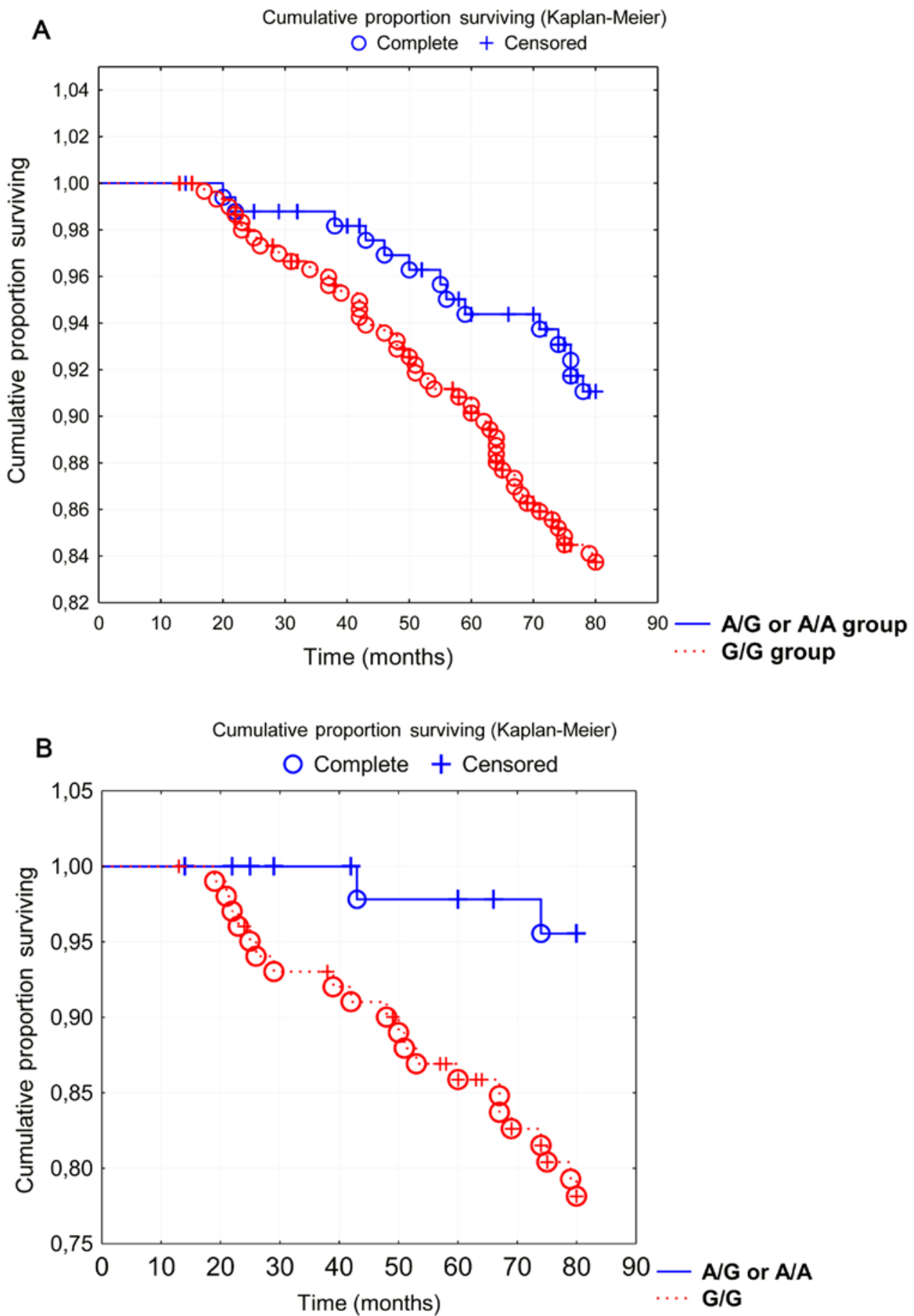

Figure 1. Cardiovascular mortality in patients with the G/G genotype vs. the A/G or the A/A genotype of CD93 during a follow-up time of 6.7 years. (A) In the total study population. $\mathrm{Z}=2.23 ; \mathrm{P}=0.026$. (B) In the group with diabetes. $\mathrm{Z}=2.63 ; \mathrm{P}=0.008$. Censored participants were those still alive at the end of the study period, or those who had died for reasons other than cardiovascular disease. Completed participants were those who had died due to cardiovascular disease.

$\chi^{2}: 6.24 ; \mathrm{P}=0.013 ;$ all-cause mortality; $\mathrm{A} / \mathrm{G}: 30 / 152=19.7 \%$; $\mathrm{G} / \mathrm{G}: 70 / 301=23.3 \%$; $\mathrm{P}=0.39$ ).

Applying a Kaplan-Meier graph of the CV mortality during the follow-up period gave significantly higher mortality in the $\mathrm{G} / \mathrm{G}$ genotype $(\mathrm{Z}=2.23 ; \mathrm{P}=0.026$ ) (Fig. $1 \mathrm{~A})$.

By applying a risk evaluation of mortality using Cox proportional hazard regression in a multivariate model, a significantly higher $\mathrm{CV}$ mortality in the G/G genotype (Table V), but not in all-cause mortality (Table VI) could be seen as compared to the $\mathrm{A} / \mathrm{G}$ genotype.
Dividing the population according to sex, a significantly increased risk for the $\mathrm{G} / \mathrm{G}$ genotype could be seen in both sexes (Table V), with a possible higher risk of the genotype among the females with a point estimate of 7.08, compared to 2.21 in the male group; however, there was a broad confidence interval, especially in the female group.

In a sub-group analysis including the group with diabetes, the same distribution among the three genotypes could be seen (A/A: 2.6\%; A/G: 30.5\%; G/G: 66.2\%). By applying the mortality data, interesting results were obtained. Regarding 
Table V. Cox proportional hazard regression analysis evaluating risk of cardiovascular mortality in the study population regarding rs2749812 of CD93.

\begin{tabular}{|c|c|c|c|c|c|c|c|c|c|}
\hline \multirow[b]{2}{*}{ Variable } & \multicolumn{3}{|c|}{ Total population } & \multicolumn{3}{|c|}{ Males } & \multicolumn{3}{|c|}{ Females } \\
\hline & HR & $95 \% \mathrm{CI}$ & P-value & HR & $95 \% \mathrm{CI}$ & P-value & HR & $95 \% \mathrm{CI}$ & P-value \\
\hline Age & 1.13 & $1.05-1.22$ & 0.001 & 1.16 & $1.06-1.28$ & 0.002 & 1.08 & $0.94-1.25$ & 0.28 \\
\hline IHD & 0.99 & $0.52-1.87$ & 0.97 & 0.76 & $0.35-1.64$ & 0.49 & 2.26 & $0.67-7.61$ & 0.19 \\
\hline Hypertension & 1.25 & $0.67-2.33$ & 0.48 & 1.23 & $0.60-2.53$ & 0.57 & 1.68 & $0.45-6.21$ & 0.44 \\
\hline Diabetes & 1.34 & $0.79-2.27$ & 0.28 & 0.98 & $0.51-1.90$ & 0.95 & 2.19 & $0.76-6.26$ & 0.15 \\
\hline NYHA III & 3.10 & $1.80-5.34$ & $<0.0001$ & 4.00 & $2.08-7.69$ & $<0.0001$ & 2.16 & $0.76-6.08$ & 0.15 \\
\hline ACEI/ARB & 1.04 & $0.58-1.87$ & 0.89 & 1.30 & $0.63-2.69$ & 0.47 & 0.99 & $0.35-2.86$ & 0.99 \\
\hline Beta blockers & 1.09 & $0.62-1.91$ & 0.76 & 1.03 & $0.51-2.06$ & 0.94 & 0.88 & $0.32-2.42$ & 0.81 \\
\hline Diuretics & 0.79 & $0.45-1.40$ & 0.42 & 0.53 & $0.25-1.10$ & 0.09 & 3.78 & $1.16-12.30$ & 0.03 \\
\hline $\mathrm{Hb}<120 \mathrm{~g} / \mathrm{l}$ & 1.04 & $0.50-2.15$ & 0.92 & 1.09 & $0.37-3.21$ & 0.88 & 3.73 & $1.08-12.84$ & 0.04 \\
\hline Rs2749812 G/G & 1.96 & $1.07-3.57$ & 0.03 & 2.21 & $1.11-4.42$ & 0.02 & 7.08 & $1.31-38.42$ & 0.02 \\
\hline
\end{tabular}

ACEI, ACE-inhibitor; ARB, angiotensin receptor blocker; IHD, ischemic heart disease; NYHA, New York Heart Association functional class; HR, hazard ratio; 95\% CI, 95\% confidence interval.

Table VI. Cox proportional hazard regression analysis evaluating risk of all-cause mortality in the study population regarding rs2749812 of CD93.

\begin{tabular}{lccc}
\hline Variable & HR & $95 \%$ CI & P-value \\
\hline Age & 1.09 & $1.03-1.16$ & 0.003 \\
IHD & 1.05 & $0.65-1.71$ & 0.84 \\
Hypertension & 1.32 & $0.81-2.15$ & 0.26 \\
Diabetes & 1.47 & $0.98-2.20$ & 0.06 \\
NYHA III & 2.97 & $1.95-4.53$ & $<0.0001$ \\
ACEI/ARB & 0.87 & $0.55-1.37$ & 0.55 \\
Beta blockers & 1.08 & $0.70-1.65$ & 0.73 \\
Diuretics & 0.93 & $0.61-1.43$ & 0.74 \\
Hb <120 g/l & 0.83 & $0.45-1.53$ & 0.54 \\
Rs2749812 G/G & 1.19 & $0.79-1.80$ & 0.41 \\
\hline
\end{tabular}

ACEI, ACE-inhibitors; ARB, angiotensin receptor blockers; IHD, ischemic heart disease; NYHA, New York Heart Association functional class; HR, hazard ratio; $95 \%$ CI, 95\% confidence interval.

CV mortality, a significant difference between the $A / G$ and the $\mathrm{G} / \mathrm{G}$ groups could be seen in those with diabetes $(\mathrm{A} / \mathrm{G}$ : 2/47=4.3\%; G/G: $\left.21 / 102=20.6 \% ; \chi^{2}: 6.58 ; \mathrm{P}=0.010\right)$; however, there was no significant difference between the two groups in all-cause mortality (A/G: 9/47=19.1\%; G/G: 32/102=31.4\%; $\chi^{2}$ : 2.41; $\mathrm{P}=0.12$ ).

Fig. 1B shows the results of applying the CV mortality data in a Kaplan-Meier graph, where there is a significant difference in mortality between the $\mathrm{G} / \mathrm{G}$ and the $\mathrm{A} / \mathrm{G}$ or $\mathrm{A} / \mathrm{A}$ groups combined.

Applying the mortality data in a multivariate risk model, where the CV mortality risk competes with several well-known clinical risk factors, the G/G group still showed an increased risk. The point estimate was high, HR: 5.2; however, as the
Table VII. Cox proportional hazard regression analysis evaluating risk of cardiovascular mortality in the study population with diabetes regarding rs2749812 of CD93.

\begin{tabular}{lccc}
\hline & \multicolumn{3}{c}{ Population with diabetes } \\
\cline { 2 - 4 } Variable & HR & $95 \%$ CI & P-value \\
\hline Age & 1.14 & $0.99-1.30$ & 0.07 \\
\hline IHD & 1.92 & $0.63-5.82$ & 0.25 \\
Hypertension & 1.37 & $0.52-3.62$ & 0.52 \\
AECI/ARB & 0.76 & $0.30-1.94$ & 0.57 \\
Beta blockers & 0.68 & $0.25-1.91$ & 0.47 \\
Diuretics & 0.74 & $0.28-1.96$ & 0.54 \\
Hb <120 g/1 & 1.79 & $0.63-5.13$ & 0.28 \\
NYHA III & 2.29 & $0.89-5.86$ & 0.08 \\
CD93 rs2749812 G/G & 5.20 & $1.20-22.62$ & 0.028
\end{tabular}

ACEI, angiotensin converting enzyme inhibitors; ARB, angiotensin receptor blockers; EF, ejection fraction; NYHA, New York Heart Association functional class III; HR, hazard ratio; 95\% CI, 95\% confidence interval.

confidence interval was broad, the information should be interpreted with caution (Table VII).

\section{Discussion}

In this study, significant differences could be demonstrated in $\mathrm{CV}$ mortality between the $\mathrm{G} / \mathrm{G}$ and the $\mathrm{A} / \mathrm{G}$ genotypes, with a higher risk in the $\mathrm{G} / \mathrm{G}$ group. It is interesting to note that in the study population an elderly community-living group of persons were investigated, that is, all those aged 70 to 85 were invited, and almost seven years of follow-up were applied. 
Even if the median age at inclusion of the participants was high, 77 years, significant differences in mortality could be demonstrated in the G/G genotype.

Also, in the female group compared to the male group, a tendency for a higher point estimate of the cardiovascular risk by having the $\mathrm{G} / \mathrm{G}$ genotype could be demonstrated. However, the sample size was limited, and the confidence interval was wide, so we interpret the result as indicating that both sexes having the $\mathrm{G} / \mathrm{G}$ genotype are exposed to a greater cardiovascular risk, and the females might be exposed to the highest risk.

As patients with diagnosed diabetes are exposed to a higher mortality risk in general, due to the diagnosis, the group with diabetes was examined in a sub-group analysis. From the results it could be seen that in this patient group the G/G genotype group were also exposed to a higher cardiovascular risk compared to the $\mathrm{A} / \mathrm{G}$ group. No statistical evaluations of the A/A group were performed, as the results would have suffered from a high degree of uncertainty due to the small sample size. Taking into consideration that the evaluation was performed in an elderly patient group, where well-known diseases influencing mortality had already occurred, the significant differences in mortality between the genotypes could indicate that this effect still has important prognostic information.

In the evaluation of a potential association between the plasma concentration of CD93 and clinical conditions and mortality, it could be demonstrated that in those with a higher plasma concentration, a higher proportion of patients with hypertension was found. Also, a higher proportion of patients on treatment with ACE inhibitors/angiotensin receptor blockers could be demonstrated in those with the higher plasma concentration of CD93. This could imply that the same diseased population was treated with pharmacotherapy. However, no difference in beta blocker treatment between the two groups was demonstrated.

Finally, the mortality evaluations demonstrated a higher all-cause mortality in those with a high concentration of CD93. In this study, no statistical difference could be seen regarding $\mathrm{CV}$ mortality; however, this could be an effect of the sample size being too small to demonstrate a difference in this variable. It could also indicate that besides the fact that it appears that those with a higher plasma concentration of CD93 seem to have more hypertension and poorer renal function, the end-result appears to be increased mortality, which is interesting as this has not been reported in the literature before.

The previously mentioned report on an association between CD93 and IHD (15) is based on SNPs of CD93 that are not identical to the one evaluated in this report, rs2749812. However, Mälarstig et al (17) were able to report that those with the A allele of the same SNP exhibited a higher expression of mRNA and the soluble form of CD93. This concurs with the new and interesting results that we have presented in this study. rs2749812 is located in exon 2 of CD93 and the mechanisms of how the different genotypes affect mRNA expression and soluble CD93 protein are currently not known.

There can be arguments against the reliability of the causes of death based on death certificates, as only $9.8 \%$ of the deaths were followed by an autopsy. However a recent study demonstrated a $55 \%$ accuracy of death certificates stating cardiovascular diseases as a mortality reason in a Swedish community (20). Therefore, we believe that it is possible in this report to draw conclusions on those who suffered cardiovascular mortality.

To validate the mortality results obtained, we applied a three-step statistical procedure; after the Student t-tests, we applied Kaplan-Meier evaluations, and finally a Cox proportional hazard regression was employed. All evaluations indicated results in the same direction, so we argue that in spite of the small sample size the mortality information is correct.

From the literature it could be found that CD93 is shed from neutrophils and monocytes during increased inflammatory activity (2). In this study, those that demonstrated an increased plasma concentration also had conditions with increased inflammatory activity (hypertension, impaired renal function); thus, the previous report concurs with the results obtained here. In the literature, it has been suggested that CD93 could be regarded as a candidate gene for atherosclerosis based on its immunological activity $(17,21)$. Therefore, as health resources are increasingly under pressure and society is calling for increased cost efficiency, the use of biomarkers that could identify those at risk, and maybe even more important, those at less risk, could be a way for the clinician to optimise the individual handling of patients. Here evaluations of genotypes, like those in CD93, could be an alternative, as indicated from our results.

However, future research will be needed to discover whether this glycoprotein could be one of the multitude of potential biomarkers to identify risk patients for cardiovascular disease and mortality.

The present study is based on a small study sample, so the obtained results (point estimates, confidence intervals) should be interpreted with caution. However, by applying different types of evaluations, all pointing in the same direction, it seems that the results add new and interesting information.

The study population had a limited age stratum, and were already elderly at inclusion. This could limit the interpretation into other age strata. The demonstrated data are still of interest, even though it would have been even more interesting to include a larger study sample with a greater age span.

Finally, the included population consisted of a homogenous Caucasian population, so we do not have information regarding other populations.

In conclusion, in a healthy community-living population of elderly persons, the plasma concentration of CD93 was evaluated in relation to co-morbidities and mortality during a follow-up period of 6.7 years. Those with the highest quartile demonstrated a higher all-cause mortality, as compared to those with a lower plasma concentration.

Evaluating the genotypes A/A, A/G and G/G of rs2749812 of CD93 revealed a higher CV mortality in the G/G genotype, which could be confirmed by risk evaluation using Cox regressions. It seems that the female group demonstrated more obvious genotype differences compared to the males.

Also, those with diabetes showed even greater differences, indicating a higher risk for those with the $\mathrm{G} / \mathrm{G}$ genotype.

Therefore, the data indicate that CD93 could be used in clinical situations in order to optimise the handling of patients with ischaemic heart disease.

However, the obtained data, even though interesting, should be regarded as hypothesis-generating, as the sample size was limited, and thus interpretation should be carried out 
with caution. It seems that more research in this interesting field is needed.

\section{Acknowledgements}

Not applicable.

\section{Funding}

The present study was supported by grants from the County Council of Östergötland, University of Linköping, Linköping, Sweden, and the Swedish Heart and Lung Foundation.

\section{Availability of data and materials}

The datasets generated and/or analyzed during the current study are not publicly available due to that under Swedish Law, the authors cannot share the data underlying this study and cannot do any further research than what is specified in the ethical permissions application. For inquires on the data, researchers should first reach out to the owner of the database, the University of Linköping. Please reach out to the corresponding author with requests and for assistance with data requests. If the university approves the request, researchers can submit an application to the Regional Ethical Review Board for the specific research question that the researcher wants to examine.

\section{Authors' contributions}

UA and DW conceived and designed the experiments. UA, LS and DW performed the experiments. UA and DW analyzed the data. DW contributed reagents/material/analysis tools. UA and DW wrote the manuscript. All authors read and approved final manuscript.

\section{Ethics approval and consent to participate}

All participants gave their written informed consent, and have given written permission for publication of obtained data, and the study was conducted in accordance with the Declaration of Helsinki principles. The study protocol was approved by the Regional Ethical Review Board of Linköping, Sweden (Dnr 95044).

\section{Patient consent for publication}

All participants have given their written permission for publication of obtained data.

\section{Competing interests}

The authors declare that they have no competing interests.

\section{References}

1. Bohlson SS, Silva R, Fonseca MI and Tenner AJ: CD93 is rapidly shed from the surface of human myeloid cells and the soluble form is detected in human plasma. J Immunol 175: 1239-1247, 2005.

2. Greenlee MC, Sullivan SA and Bohlson SS: Detection and characterization of soluble CD93 released during inflammation. Inflamm Res 58: 909-919, 2009.
3. Jeon JW, Jung JG, Shin EC, Choi HI, Kim HY, Cho ML, Kim SW, Jang YS, Sohn MH, Moon JH, et al: Soluble CD93 induces differentiation of monocytes and enhances TLR responses. J Immunol 185: 4921-4927, 2010.

4. Park HJ, Han H, Lee SC, Son YW, Sim DW, Park KH, Park YH, Jeong KY, Park JW and Lee JH: Soluble CD93 in serum as a marker of allergic inflammation. Yonsei Med J 58: 598-603, 2017.

5. Sigari N, Jalili A, Mahdawi L, Ghaderi E and Shilan M: Soluble CD93 as a novel biomarker in asthma exacerbation. Allergy Asthma Immunol Res 8: 461-465, 2016.

6. Duvetorp A, Slind Olsen R, Skarstedt M, Söderman J and Seifert O: Psoriasis and pro-angiogenetic factor CD93: Gene expression and association with gene polymorphism suggests a role in disease pathogenesis. Acta Derm Venereol 97: 916-921, 2017.

7. Olsen RS, Lindh M, Vorkapic E, Andersson RE, Zar N, Löfgren S, Dimberg J, Matussek A and Wågsäter D: CD93 gene polymorphism is associated with disseminated colorectal cancer. Int J Colorectal Dis 30: 883-890, 2015.

8. Borah S, Vasudevan D and Swain RK: C-type lectin family XIV members and angiogenesis. Oncol Lett 18: 3954-3962, 2019.

9. Khan KA, McMurray JL, Mohammed F and Bicknell R: C-type lectin domain group 14 proteins in vascular biology, cancer and inflammation. FEBS J 286: 3299-3332, 2019.

10. van der Ende MY, Hartman MH, Hagemeijer Y, Meems LM, de Vries HS, Stolk RP, de Boer RA, Sijtsma A, van der Meer P, Rienstra $M$ and van der Harst P: The lifelines cohort study: Prevalence and treatment of cardiovascular disease and risk factors. Int J Cardiol 228: 495-500, 2017.

11. Nanchen D, Klingenberg R, Gencer B, Räber L, Carballo D, von Eckardstein A, Windecker S, Rodondi N, Lüscher TF, Mach F, et al: Inflammation during acute coronary syndromes-risk of cardiovascular events and bleeding. Int J Cardiol 287: 13-18, 2019.

12. Golia E, Limongelli G, Natale F, Fimiani F, Maddaloni V, Pariggiano I, Bianchi R, Crisci M, D'Acierno L, Giordano R, et al: Inflammation and cardiovascular disease: From pathogenesis to therapeutic target. Curr Atheroscler Rep 16: 435, 2014.

13. Ndrepepa G: Myeloperoxidase-A bridge linking inflammation and oxidative stress with cardiovascular disease. Clin Chim Acta 493: 36-51, 2019.

14. Kaplan RC and Frishman WH: Systemic inflammation as a cardiovascular disease risk factor and as a potential target for drug therapy. Heart Dis 3: 326-332, 2001.

15. van der Net JB, Oosterveer DM, Versmissen J, Defesche JC, Yazdanpanah M, Aouizerat BE, Steyerberg EW, Malloy MJ, Pullinger CR, Kastelein JJ, et al: Replication study of 10 genetic polymorphisms associated with coronary heart disease in a specific high-risk population with familial hypercholesterolemia. Eur Heart J 29: 2195-2201, 2008.

16. Youn JC, Yu HT, Jeon JW, Lee HS, Jang Y, Park YW, Park YB, Shin EC and Ha JW: Soluble CD93 levels in patients with acute myocardial infarction and its implication on clinical outcome. PLoS One 9: e96538, 2014.

17. Mälarstig A, Silveira A, Wågsäter D, Öhrvik J, Bäcklund A, Samnegărd $\mathrm{A}$, Khademi $\mathrm{M}$, Hellenius $\mathrm{ML}$, Leander $\mathrm{K}$, Olsson T, et al: Plasma CD93 concentration is a potential novel biomarker for coronary artery disease. J Intern Med 270: 229-236, 2011.

18. Alehagen U, Ericsson A and Dahlström U: Are there any significant differences between females and males in the management of heart failure? Gender aspects of an elderly population with symptoms associated with heart failure. J Card Fail 15: 501-507, 2009.

19. Alehagen U and Wågsäter D: Gender difference and genetic variance in lipoprotein receptor-related protein 1 is associated with mortality. Biomed Rep 1: 1-5, 2019.

20. Eriksson A, Stenlund H, Ahlm K, Boman K, Bygren LO, Johansson LA, Olofsson BO, Wall S and Weinehall L: Accuracy of death certificates of cardiovascular disease in a community intervention in Sweden. Scand J Public Health 41: 883-889, 2013.

21. Greenlee MC, Sullivan SA and Bohlson SS: CD93 and related family members: Their role in innate immunity. Curr Drug Targets 9: 130-138, 2008.

This work is licensed under a Creative Commons Attribution-NonCommercial-NoDerivatives 4.0 International (CC BY-NC-ND 4.0) License. 